\title{
ALMOST SURE STABLE OSCILLATIONS IN A LARGE SYSTEM OF RANDOMLY COUPLED EQUATIONS*
}

\author{
STUART GEMAN $\dagger$
}

\begin{abstract}
This paper is about limiting (large system) behavior of a set of differential equations with random coefficients. Under certain conditions the behavior of the entire system is well described by a small number of "prototype" equations, and these can be derived, heuristically, by applying a law of large numbers to the original system. An application of this theorem is the specification of a small number of parameters which guarantee that sufficiently large versions of the systems studied will oscillate with a predicted period and wave form.
\end{abstract}

1. Introduction. This paper continues a study of limit laws for large systems of weakly and randomly coupled equations, begun in [5]. In many instances, the correct limiting (large system) behavior of the solutions of these equations can be guessed from an assumption of "local chaos": roughly, the assumption that the solutions of individual equations within the system are mutually independent. A consequence of this assumption will often be a specific conjecture about the limiting behavior of the system. Although the solutions are not, in fact, independent, intuition suggests that they are "nearly" so, and the resulting conjecture is quite often correct.

The main result of this paper is about the behavior of a system of nonlinear differential equations consisting of large "homogeneous" subpopulations. The coefficients which couple these equations are random variables. The subpopulations are homogeneous in the sense that these random variables have a common distribution within each subpopulation. I will make an assumption of local chaos and derive its consequence, which is that the behavior of the entire system can be described arbitrarily well, as the number of equations grows to infinity, by the solutions to a small set of deterministic equations, one for each subpopulation. The conjecture, then, is a sort of law of large numbers for the system of differential equations; it is the purpose of this paper to prove that conjecture. If there are two subpopulations, then the associated deterministic equations form a two-dimensional system, and parameters of the full system can be chosen so that this reduced system has a globally stable limit cycle. By the theorem we present, the entire system must demonstrate a stable oscillation, with period and wave form predicted by the two-dimensional equation, provided that the full system is sufficiently large. Computer simulation nicely illustrates this application of the theorem.

The theorem is about a system of differential equations containing a relatively "simple" nonlinearity. In this sense, the result is a natural first extension of the results obtained in [5] about purely linear systems. The motivation for this particular set of equations is that they are essentially those used in [6] to model the activities of brainstem respiratory neurons. In that article, we argued intuitively for the reduction of the full set of random equations to a small set of deterministic equations, and used the reduced equations to study the behavior of the full system. The analytic results presented here, together with numerous computer simulations of related systems (see, for example, [1]), make it clear that such approximations are broadly applicable. Amari, in [1], first argued that this would indeed be the case; the study here, like the one in [6], was strongly influenced by his reasoning.

* Received by the editors March 11, 1981. This work was supported by the National Science Foundation under grant MCS76-80762.

$\dagger$ Division of Applied Mathematics, Brown University, Providence, Rhode Island 02912. 
2. Main result. The theorem is best introduced by first considering a special case:

$$
\frac{d}{d t} x_{i}(t)=-\alpha x_{i}(t)+\frac{1}{n} \sum_{j=1}^{n} w_{i j} f\left(x_{j}(t)\right), \quad 1 \leqq i \leqq n,
$$

where

1. the initial conditions, $x_{1}(0), \cdots, x_{n}(0)$, are arbitrary,

2. $\alpha$ is an arbitrary positive constant,

3. $f(x)$ is a bounded and uniformly Lipschitz continuous function from $\boldsymbol{R}^{1}$ to $\boldsymbol{R}^{1}$, and

4. $\left\{w_{i j}\right\} i=1,2, \cdots, j=1,2, \cdots$ are independent and identically distributed (i.i.d.) random variables.

A further assumption on the random variables $\left\{w_{i j}\right\}$ will be added later. (Actually, $x_{i}(t)$, and possibly the initial condition, $x_{i}(0)$, depends on $n$, but I will not explicitly indicate this dependence.)

Although the mathematical problem will be clear without any understanding of the biology, the reader may be interested in the connection to neural modelling. Equations like (2.1) have been widely used to describe the dynamics of interconnected networks of neural-like elements (for some examples, see [1], [3], [7]). $x_{i}(t)$ represents the membrane potential, at the cell body, of the $i$ th neuron at time $t$. In the absence of input to this $i$ th cell, the potential is assumed to decay exponentially to 0 (the arbitrarily chosen "resting potential"), with a time constant $1 / \alpha . f\left(x_{j}(t)\right)$ models the conversion of the $j$ cell body membrane potential to frequency of action potentials transmitted along the $j$ cell axon. The precise form of $f$ is unimportant (for our purposes), but evidence suggests a positive, bounded, and increasing function. The activity in the axon of the $j$ cell effects a change in membrane potential at the $i$ th cell through a "synaptic connection". The strength of this connection is represented by $w_{i j} / n$. (" $1 / n$ " because it is assumed that the total input to the $i$ cell remains "order 1 ", no matter how large $n$ becomes.) $w_{i j}$ may be positive or negative, modelling, respectively, an "excitatory" or an "inhibitory" $j$ to $i$ synapse. When there is no $j$ to $i$ synapse, $w_{i j}$ is zero. It is assumed that the combined influence on the $i$ cell membrane potential of inputs from all other cells in the network is additive: hence, the term $\sum_{j=1}^{n} w_{i j} f\left(x_{j}(t)\right)$. In [6], Miller and I have argued for a model of the brainstem respiratory centers consisting of four interconnected subpopulations of modelled neurons, with each subpopulation of essentially the type described in (2.1).

What can be said about the behavior of this system when $n$ is large? It would seem that in a large system a knowledge of any one of the random variables $x_{i}(t)$ would contribute very little information about the value of any of the other $x_{j}(t), j \neq i$, or about any of the random variables $\left\{w_{i j}\right\}$. This leads to a "chaos hypothesis": when $n$ is large, the system behaves as if the random variables $x_{1}(t), \cdots, x_{n}(t)$ were independent of each other and of the random variables $\left\{w_{i j}\right\}$. If we pretend that all of these variables are in fact independent (certainly, they are not), then we can guess exactly the asymptotic (large $n$ ) behavior: By the law of large numbers

$$
\begin{aligned}
& \frac{1}{n} \sum_{j=1}^{n} w_{i j} f\left(x_{j}(t)\right) \approx E\left[w_{11}\right] \frac{1}{n} \sum_{j=1}^{n} E\left[f\left(x_{j}(t)\right)\right] \\
& \Rightarrow \frac{d}{d t} x_{i}(t) \approx-\alpha x_{i}(t)+E\left[w_{11}\right] \frac{1}{n} \sum_{j=1}^{n} E\left[f\left(x_{j}(t)\right)\right] \quad \forall i \\
& \quad \Rightarrow \frac{d}{d t}\left(x_{i}(t)-x_{i^{\prime}}(t)\right) \approx-\alpha\left(x_{i}(t)-x_{i^{\prime}}(t)\right) \quad \forall i, i^{\prime}
\end{aligned}
$$




$$
\begin{aligned}
\Rightarrow & \varlimsup_{t \rightarrow \infty}\left|x_{i}(t)-x_{i^{\prime}}(t)\right| \approx 0 \quad \forall i, i^{\prime} \\
\Rightarrow & (\operatorname{put}(2.3) \text { into }(2.2)) \\
& \frac{d}{d t} x_{i}(t) \approx-\alpha x_{i}(t)+E\left[w_{11}\right] E\left[f\left(x_{i}(t)\right)\right] \quad \text { (for } t \text { large) } \\
\Rightarrow & \frac{d}{d t} x_{i}(t) \approx-\alpha x_{i}(t)+E\left[w_{11}\right] f\left(x_{i}(t)\right) \quad(\text { for } t \text { large) }
\end{aligned}
$$

the last implication because $x_{i}(t)$ should be nearly deterministic (suggested by (2.4)), i.e., the support of its distribution should concentrate near a single point. We are led, then, to conjecture that for large $n$

$$
\varlimsup_{t \rightarrow \infty}\left|x_{i}(t)-x_{i^{\prime}}(t)\right|
$$

and

$$
\varlimsup_{t \rightarrow \infty}\left|\frac{d}{d t} x_{i}(t)-\left\{-\alpha x_{i}(t)+E\left[w_{11}\right] f\left(x_{i}(t)\right)\right\}\right|
$$

are small for all $i$ and $i^{\prime}$. In fact, as the theorem shows (with one further condition on $\left.\left\{w_{i j}\right\}\right)$ :

and

$$
\lim _{n \rightarrow \infty} \varlimsup_{t \rightarrow \infty} \sup _{1 \leqq i, i^{\prime} \leqq n}\left|x_{i}(t)-x_{i^{\prime}}(t)\right|=0 \quad \text { a.s. }
$$

$$
\lim _{n \rightarrow \infty} \varlimsup_{t \rightarrow \infty} \sup _{1 \leqq i \leqq n}\left|\frac{d}{d t} x_{i}(t)-\left\{-\alpha x_{i}(t)+E\left[w_{11}\right] f\left(x_{i}(t)\right)\right\}\right|=0 \quad \text { a.s. }
$$

The theorem is about a slight generalization: it is about $P$ interconnected systems of equations, each of the form of (2.1). For $k=1,2, \cdots, P, n_{k}$ will denote the number of equations in the $k$ th system, and $x_{i}^{k}(t) 1 \leqq i \leqq n_{k}$ will denote the $i$ th dependent variable in the $k$ th system. Each system may have its own characteristic "output function" $f^{k}(\cdot)$, and its own decay coefficient $\alpha^{k} . x_{j}^{l}(t)$ influences $x_{i}^{k}(t)$ through its output function, $f^{l}\left(x_{j}^{l}(t)\right)$, and a "coupling constant" (which will be a random variable) $w_{i j}^{k l} / n_{l}$. Thus

$$
\frac{d}{d t} x_{i}^{k}(t)=-\alpha^{k} x_{i}^{k}(t)+\sum_{l=1}^{P} \frac{1}{n_{l}} \sum_{j=1}^{n_{l}} w_{i j}^{k l} f\left(x_{j}^{l}(t)\right), \quad 1 \leqq k \leqq P, \quad 1 \leqq i \leqq n_{k} .
$$

The initial conditions, $x_{i}^{k}(0), k=1,2, \cdots, P, 1 \leqq i \leqq n_{k}$, are arbitrary, and $\alpha^{1}, \cdots, \alpha^{P}$ are strictly positive but otherwise arbitrary constants. The assumptions for the theorem are:

A1. For each $k=1,2, \cdots, P, f^{k}(\cdot)$ is a bounded and uniformly Lipschitz continuous function.

A2. For each $k=1,2, \cdots, P$ and $l=1,2, \cdots, P, w_{i j}^{k l}, i=1,2, \cdots, j=1,2, \cdots$, is an (infinite) collection of i.i.d. random variables with $E\left[e^{i \lambda w_{11}^{k l}}\right]$ analytic at $\lambda=0$. And, a condition relating the growths of the subpopulations:

A3. For each $k=1,2, \cdots, P, n_{k}=n_{k}(n)$ is a sequence of integers, indexed by $n$, satisfying

$$
\lim _{n \rightarrow \infty} \frac{n_{k}(n)}{n}=c_{k}
$$

for some constant $c_{k}>0$. 
THEOREM. Under the assumptions $\mathrm{A} 1, \mathrm{~A} 2$, and $\mathrm{A} 3$,

$$
\lim _{n \rightarrow \infty} \varlimsup_{t \rightarrow \infty} \sup _{k=1, \cdots, P} \sup _{1 \leqq i, i^{\prime} \leqq n_{k}}\left|x_{i}^{k}(t)-x_{i^{\prime}}^{k}(t)\right|=0 \quad \text { a.s. }
$$

and

$$
\lim _{n \rightarrow \infty} \varlimsup_{t \rightarrow \infty} \sup _{k=1, \cdots, P} \sup _{1 \leqq i \leqq n_{\min }}\left|\frac{d}{d t} x_{i}^{k}(t)-\left\{-\alpha x_{i}^{k}(t)+\sum_{l=1}^{P} E\left[w_{11}^{k l}\right] f^{l}\left(x_{i}^{l}(t)\right)\right\}\right|=0 \quad \text { a.s., }
$$

where $n_{\min }=\min \left(n_{1}, \cdots, n_{p}\right)$.

Loosely put, the entire system is arbitrarily well represented by a system of $P$ prototype equations:

$$
\frac{d}{d t} x^{k}(t)=-\alpha^{k} x^{k}(t)+\sum_{l=1}^{P} E\left[w_{11}^{k l}\right] f^{l}\left(x^{l}(t)\right)
$$

$k=1,2, \cdots, P$. A good illustration is the case $P=2$, in which parameters are chosen so that the prototype equations,

$$
\begin{aligned}
& \frac{d}{d t} x^{1}(t)=-\alpha^{1} x^{1}(t)+E\left[w_{11}^{11}\right] f^{1}\left(x^{1}(t)\right)+E\left[w_{11}^{12}\right] f^{2}\left(x^{2}(t)\right) \\
& \frac{d}{d t} x^{2}(t)=-\alpha^{2} x^{2}(t)+E\left[w_{11}^{21}\right] f^{1}\left(x^{1}(t)\right)+E\left[w_{11}^{22}\right] f^{2}\left(x^{2}(t)\right),
\end{aligned}
$$

have a globally stable limit cycle. In fact, if (for example)

$$
\begin{aligned}
& \alpha^{1}=5, \quad \nexists=1, \quad E\left[w_{11}^{11}\right]=12, \quad E\left[w_{11}^{12}\right]=13, \\
& E\left[w_{11}^{21}\right]=4, \quad E\left[w_{11}^{22}\right]=5, \\
& f^{1}(x)=f^{2}(x)=\tan ^{-1}(x),
\end{aligned}
$$

then (2.5) does have such a limit cycle. (The system is bounded, the only equilibrium point $\left(x^{1}=x^{2}=0\right)$ is unstable, and there is only one periodic orbit.) The dotted curves in Fig. 1 are phase portraits of the limit cycle for (2.5).

Take $n_{1}=n_{2}=n$ and $\left\{w_{i j}^{11}\right\},\left\{w_{i j}^{12}\right\},\left\{w_{i j}^{21}\right\},\left\{w_{i j}^{22}\right\}$ all Gaussian with variance 50 and means as specified in (2.6). Notice that for all of the random coefficients the standard deviation exceeds $50 \%$ of the mean; this is not merely a perturbation on the trivial case in which all coefficients of a given type (e.g., all $\left.w_{i j}^{11}, 1 \leqq i, j \leqq n\right)$ are identical. The theorem says that when $n$ is large, all of the variables $x_{1}^{1}(t), \cdots, x_{n}^{1}(t)$ will eventually be close together, and will remain close together as $t \rightarrow \infty$. The same is true of $x_{1}^{2}(t), \cdots, x_{n}^{2}(t)$, and, furthermore, the large $t$ phase portrait for any pair, $\left(x_{i}^{1}(t), x_{i}^{2}(t)\right), 1 \leqq i \leqq n$, will approximate (arbitrarily well as $\left.n \rightarrow \infty\right)$ the phase portrait of (2.5). Hence, in a large version of the present system we expect oscillations which are well described by the limit cycle for (2.5). (The precise statement is somewhat tedious, but it does follow easily from the statement of the theorem. Roughly it is that for any $\varepsilon>0$, there exists (almost surely) an $n_{0}$ such that for all $n \geqq n_{o}$ : (1) the (Euclidean) distance between any two of the pairs, $\left(x_{i}^{1}(t), x_{i}^{2}(x)\right)$ and $\left(x_{u_{i}}^{1},(t), x_{u_{i}}^{2}\right.$, $(t))$, will eventually become and remain less than $\varepsilon ;(2)$ all pairs $\left(x_{i}^{1}(t), x_{i}^{2}(t)\right)$ will eventually enter and remain inside of the annulus defined by the $\varepsilon$ neighborhood of the limit cycle for (2.5), and (3) each pair will circle that region in the same direction as the limit cycle of (2.5), and with a period that is within $\varepsilon$ of the limit cycle period.)

In a typical experiment (see Fig. 1), when $n$ was smaller than 7 all variables approached equilibrium values. With $n=7$, the system oscillated, but these oscillations 


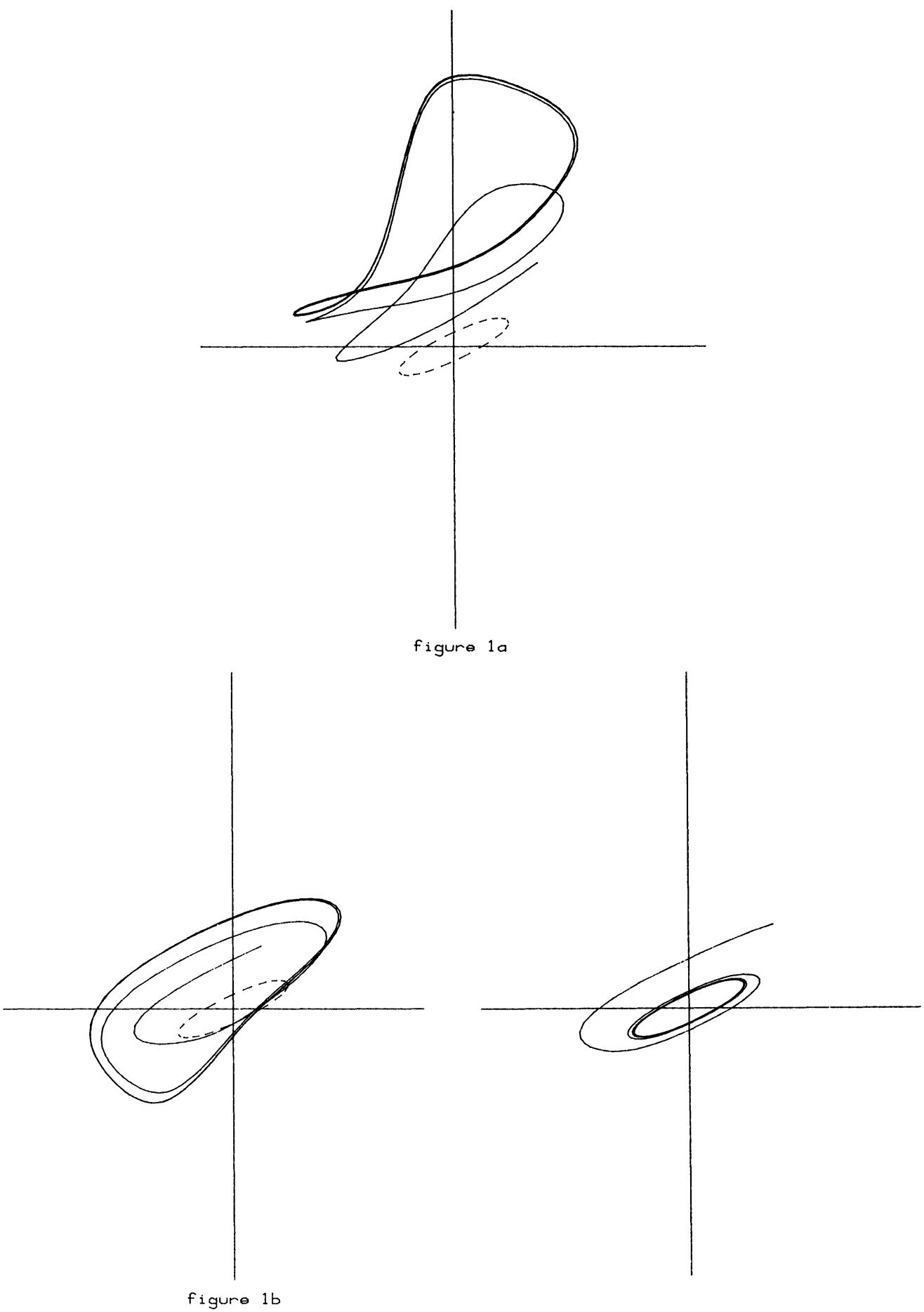

FIG 1. (a) Dotted curve is the large $t$ (limit cycle) trajectory for $\left(x^{1}(t), x^{2}(t)\right)$ in (2.5), the "prototype" equation. Solid curve is $\left(x_{1}^{1}(t), x_{1}^{2}(t)\right)$ trajectory when $n=7$. (b) Dotted curve is as in a). Solid curve is trajectory of the population averages, $\left((1 / n) \sum_{i=1}^{n} x_{i}^{1}(t),(1 / n) \sum_{i=1}^{n} x_{i}^{2}(t)\right)$, when $n=7$. (c) Trajectory of the population averages when $n=80$. The large t trajectory is indistinguishable from the limit cycle of the prototype system, (2.5). (The large t trajectory of $\left(x_{1}^{1}(t), x_{1}^{2}(t)\right)$ is not noticeably different.) All three figures use the same scale. 
did not resemble the behavior of the prototype system, (2.5) (Fig. 1, a and b). When $n$ equalled 80, the theorem was in full force: the large $t$ behavior of the random system was virtually indistinguishable from that of the prototype system (Fig. 1, c).

3. Proof of the Theorem. Everything involved in proving the theorem is already illustrated in the special case $P=1$ (i.e., (2.1)). I will show that, in (2.1),

$$
\lim _{n \rightarrow \infty} \varlimsup_{t \rightarrow \infty} \sup _{1 \leqq i, i^{\prime} \leqq n}\left|x_{i}(t)-x_{i^{\prime}}(t)\right|=0 \quad \text { a.s. }
$$

and

$$
\lim _{n \rightarrow \infty} \varlimsup_{t \rightarrow \infty} \sup _{1 \leqq i \leqq n}\left|\frac{d}{d t} x_{i}(t)-\left\{-\alpha x_{i}(t)+E\left[w_{11}\right] f\left(x_{i}(t)\right)\right\}\right|=0 \quad \text { a.s. }
$$

provided that $E\left[e^{i \lambda w_{11}}\right]$ is analytic at $\lambda=0$. The proof will make use of the following notation:

$W=\left\{w_{i j}\right\}, 1 \leqq i, j \leqq n$, the $n \times n$ matrix whose $(i, j)$ component is $w_{i j}$.

$m=E\left[w_{11}\right]$.

$M=\{m\}$, the $n \times n$ matrix with all components $m$.

$X(t)=\left(x_{1}(t), \cdots, x_{n}(t)\right)^{T}(T$ denotes transpose $)$.

$F(X(t))=\left(f\left(x_{1}(t)\right), \cdots, f\left(x_{n}(t)\right)\right)^{T}$.

$\tilde{X}(t)=(1 / n) M \int_{0}^{t} e^{-\alpha(t-s)} F(X(s)) d s$ (define the integral of a vector componentwise).

$\tilde{x}_{i}(t)=i$ th component of $\tilde{X}(t), 1 \leqq i \leqq n$. Notice that all $\tilde{x}_{i}(t)$ are identical and equal to

$$
\frac{m}{n} \sum_{j=1}^{n} \int_{0}^{t} e^{-\alpha(t-s)} f\left(x_{j}(s)\right) d s
$$

$F(\tilde{X}(t))=\left(f\left(\tilde{x}_{1}(t)\right), \cdots, f\left(\tilde{x}_{n}(t)\right)\right)^{T}$.

$\|V\|$ is the operator ("induced") norm when $V$ is a matrix. It is the Euclidean norm when $V$ is a vector.

A final preliminary:

LEMMA. If $v_{i j} i=1,2, \cdots, j=1,2, \cdots$ are i.i.d. random variables with common mean $u$, and if $E\left[e^{i \lambda v_{11}}\right]$ is analytic at $\lambda=0$, then

$$
\lim _{n \rightarrow \infty} \sup _{1 \leqq i \leqq n}\left|\frac{1}{n} \sum_{j=1}^{n} v_{i j}-u\right|=0 \quad \text { a.s. }
$$

Proof. Fix $\varepsilon>0$.

$$
P\left(\sup _{1 \leqq i \leqq n}\left|\frac{1}{n} \sum_{j=1}^{n} v_{i j}-u\right|>\varepsilon\right) \leqq n P\left(\left|\frac{1}{n} \sum_{j=1}^{n} v_{1 j}-u\right|>\varepsilon\right) \leqq 2 n e^{-n \delta},
$$

for some $\delta>0$. (The last step is a typical large deviation result; see, for example, Chernoff [2].) Now apply the Borel-Cantelli lemma, and conclude (3.3).

The plan of the proof of the theorem is to first show that $X(t)$ is close to $\tilde{X}(t)$. Since the components of the latter are all the same, (3.1) follows. (3.2) then comes from putting (3.1) back into the original equation, (2.1).

Start with a preliminary bound on $\|X(t)-\tilde{X}(t)\|\left(\right.$ let $\left.\beta=\sup _{x \in R}|f(x)|\right)$ :

$$
X(t)-\tilde{X}(t)=X(0) e^{-\alpha t}+\frac{1}{n}(W-M) \int_{0}^{t} e^{-\alpha(t-s)} F(X(s)) d s
$$




$$
\begin{aligned}
\Rightarrow \varlimsup_{n \rightarrow \infty} \varlimsup_{t \rightarrow \infty}\|X(t)-\tilde{X}(t)\| \\
\quad \leqq \varlimsup_{n \rightarrow \infty}\left\|\frac{W-M}{\sqrt{n}}\right\| \varlimsup_{n \rightarrow \infty} \varlimsup_{t \rightarrow \infty} \int_{0}^{t} e^{-\alpha(t-s)} \frac{\|F(x(s))\|}{\sqrt{n}} d s \\
\quad \leqq \frac{\beta}{\alpha} \varlimsup_{n \rightarrow \infty}\left\|\frac{W-M}{\sqrt{n}}\right\| \stackrel{\text { a.s. }}{=} \frac{2 \beta}{\alpha} \sqrt{E\left(w_{11}-m\right)^{2}}
\end{aligned}
$$

(the last equality was proved in [4]). Now rewrite the right-hand side in (3.4):

$X(t)-\tilde{X}(t)=X(0) e^{-\alpha t}+\frac{1}{n}(W-M) \int_{0}^{t} e^{-\alpha(t-s)}(F(X(s))-F(\tilde{X}(s))) d s$

$$
\begin{aligned}
+\frac{1}{n}(W-M) \int_{0}^{t} e^{-\alpha(t-s)} F(\tilde{X}(s)) d s & \\
\Rightarrow \sup _{1 \leqq i \leqq n}\left|x_{i}(t)-\tilde{x}_{i}(t)\right| \leqq & e^{-\alpha t} \sup _{1 \leqq i \leqq n}\left|x_{i}(0)\right| \\
& +\left\|\frac{W-M}{n}\right\| \int_{0}^{t} e^{-\alpha(t-s)}\|F(X(s))-F(\tilde{X}(s))\| d s \\
& +\int_{0}^{t} e^{-\alpha(t-s)}\left|f\left(\tilde{x}_{1}(s)\right)\right| d s \sup _{1 \leqq i \leqq n}\left|\frac{1}{n} \sum_{j=1}^{n} w_{i j}-m\right| .
\end{aligned}
$$

A consequence of the fact that $\sup _{t \geqq 0}\|F(X(t))-F(\tilde{X}(t))\|$ is finite (it is bounded by $2 \sqrt{n} \beta)$ is that

$$
\varlimsup_{t \rightarrow \infty} \int_{0}^{t} e^{-\alpha(t-s)}\|F(X(s))-F(\tilde{X}(s))\| d s \leqq \frac{1}{\alpha} \varlimsup_{t \rightarrow \infty}\|F(X(t))-F(\tilde{X}(t))\| .
$$

Recall that $f(x)$ is uniformly Lipschitz continuous. Take $\lambda>0$ such that $|f(x)-f(y)| \leqq$ $\lambda|x-y|$ for all $x, y \in R^{1}$. Then $\|F(X(t))-F(\tilde{X}(t))\| \leqq \lambda\|X(t)-\tilde{X}(t)\|$, and therefore

$$
\varlimsup_{t \rightarrow \infty} \int_{0}^{t} e^{-\alpha(t-s)}\|F(X(s))-F(\tilde{X}(s))\| d s \leqq \frac{\lambda}{\alpha} \varlimsup_{t \rightarrow \infty}\|X(t)-\tilde{X}(t)\|
$$

Use this in (3.6):

$$
\begin{aligned}
& \varlimsup_{t \rightarrow \infty} \sup _{1 \leqq i \leqq n}\left|x_{i}(t)-\tilde{x}_{i}(t)\right| \leqq\left\|\frac{W-M}{n}\right\| \frac{\lambda}{\alpha} \varlimsup_{t \rightarrow \infty}\|X(t)-\tilde{X}(t)\|+\frac{\beta}{\alpha} \sup _{1 \leqq i \leqq n}\left|\frac{1}{n} \sum_{j=1}^{n} w_{i j}-m\right| \\
& \Rightarrow \text { (by } 3.5) \\
& \varlimsup_{n \rightarrow \infty} \varlimsup_{t \rightarrow \infty} \sup _{1 \leqq i \leqq n}\left|x_{i}(t)-\tilde{x}_{i}(t)\right| \leqq \varlimsup_{n \rightarrow \infty}^{\text {a.s. }}\left\|\frac{W-M}{n}\right\| \frac{2 \lambda \beta}{\alpha^{2}} \sqrt{E\left(w_{11}-m\right)^{2}} \\
& +\frac{\beta}{\alpha} \varlimsup_{n \rightarrow \infty} \sup _{1 \leqq i \leqq n}\left|\frac{1}{n} \sum_{j=1}^{n} w_{i j}-m\right| .
\end{aligned}
$$

The result in [4] implies that $\|(W-M) / n\| \rightarrow 0$ almost surely, and the lemma above asserts that

as $n \rightarrow \infty$. Hence

$$
\sup _{1 \leqq i \leqq n}\left|\frac{1}{n} \sum_{j=1}^{n} w_{i j}-m\right| \rightarrow 0 \quad \text { a.s., }
$$

$$
\lim _{n \rightarrow \infty} \varlimsup_{t \rightarrow \infty} \sup _{1 \leqq i \leqq n}\left|x_{i}(t)-\tilde{x}_{i}(t)\right|=0 \quad \text { a.s. }
$$


Obviously, then,

$$
\lim _{n \rightarrow \infty} \varlimsup_{t \rightarrow \infty} \sup _{1 \leqq i, i^{\prime} \leqq n}\left|x_{i}(t)-x_{i^{\prime}}(t)\right|=0 \quad \text { a.s. }
$$

which is the first part of the theorem.

For the second part:

$$
\begin{aligned}
& \varlimsup_{t \rightarrow \infty} \sup _{1 \leqq i \leqq n}\left|\frac{d}{d t} x_{i}(t)-\left\{-\alpha x_{i}(t)+m f\left(x_{i}(t)\right)\right\}\right| \\
& \quad \leqq \varlimsup_{r \rightarrow \infty} \sup _{1 \leqq i \leqq n}\left|\frac{1}{n} \sum_{j=1}^{n}\left(w_{i j}-m\right) f\left(x_{i}(t)\right)\right|+\varlimsup_{t \rightarrow \infty} \sup _{1 \leqq i \leqq n} \frac{1}{n} \sum_{j=1}^{n}\left|w_{i j}\left(f\left(x_{j}(t)\right)-f\left(x_{i}(t)\right)\right)\right| \\
& (3.7) \quad \leqq \beta \sup _{1 \leqq i \leqq n}\left|\frac{1}{n} \sum_{j=1}^{n} w_{i j}-m\right|+\left(\sup _{1 \leqq i \leqq n} \frac{1}{n} \sum_{j=1}^{n}\left|w_{i j}\right|\right)\left(\lambda \varlimsup_{t \rightarrow \infty} \sup _{1 \leqq i, j \leqq n}\left|x_{j}(t)-x_{i}(t)\right|\right) .
\end{aligned}
$$

$\sup _{1 \leqq i \leqq n}(1 / n) \sum_{j=1}^{n}\left|w_{i j}\right|$ almost surely remains bounded as $n \rightarrow \infty$, by an application of the lemma $\left(E\left[e^{i \lambda w_{11}}\right]\right.$ analytic at $\lambda=0 \Rightarrow E\left[e^{i \lambda\left|w_{11}\right|}\right]$ analytic at $\left.\lambda=0\right)$. The lemma also implies that the first term in (3.7) converges to 0 as $n \rightarrow \infty$, almost surely. The proof is completed by applying the first part of the theorem, already established, to the remaining term in (3.7).

4. Conjecture on a weak limit. Look again at (2.1), but with $E\left[w_{11}\right]=0$. An implication of the theorem is that the "input" to $x_{i}(t)$,

$$
\frac{1}{n} \sum_{j=1}^{n} w_{i j} f\left(x_{j}(t)\right)
$$

is small when $n$ is large. Reasoning intuitively (i.e., assuming again a "local chaos"), we can expect a different normalization, $1 / n \rightarrow 1 / \sqrt{n}$, to produce a net input of "order 1 ", and therefore a nontrivial limit when $n \rightarrow \infty$. In other words, to get interesting limiting behavior when $E\left[w_{11}\right]=0$, the natural choice is to replace (2.1) by

$$
\frac{d}{d t} x_{i}(t)=-\alpha x_{i}(t)+\frac{1}{\sqrt{n}} \sum_{j=1}^{n} w_{i j} f\left(x_{j}(t)\right) .
$$

To guess the large $n$ behavior for (4.1), write

$$
x_{i}(t)=e^{-\alpha t}+\frac{1}{\sqrt{n}} \sum_{j=1}^{n} w_{i j} \int_{0}^{t} e^{-\alpha(t-u)} f\left(x_{j}(u)\right) d u
$$

(taking, for convenience, $x_{i}(0)=1$ for all $i$ ). The central limit theorem (with a chaos hypothesis) suggests that $x_{i}(t)$ will approach a Gaussian process, as $n \rightarrow \infty$. And, at least for any fixed $p$, we should expect the processes, $x_{1}(\cdot), \cdots, x_{p}(\cdot)$, to be asymptotically (large $n$ ) independent. If all of this is true, then the asymptotic distribution for any collection of $x$ 's is determined completely by the limiting mean and correlation functions of the individual processes, $x_{i}(t), i=1, \cdots, n$. A conjecture for the mean function, $\mu(t)=E\left[x_{i}(t)\right]$, comes from

$$
\begin{aligned}
\mu(t) & =e^{-\alpha t}+\frac{1}{\sqrt{n}} \sum_{j=1}^{n} E\left[w_{i j} \int_{0}^{t} e^{-\alpha(t-u)} f\left(x_{j}(u)\right) d u\right] \\
& \approx e^{-\alpha t}+\frac{1}{\sqrt{n}} \sum_{j=1}^{n} E\left[w_{i j}\right] E\left[\int_{0}^{t} e^{-\alpha(t-u)} f\left(x_{j}(u)\right) d u\right] \\
& =e^{-\alpha t}
\end{aligned}
$$


And, a conjecture for the correlation, $r(t, s)=E\left[x_{i}(t) x_{i}(s)\right]$, comes from

$$
\begin{aligned}
r(t, s)= & e^{-\alpha(t+s)}+e^{-\alpha s} \frac{1}{\sqrt{n}} \sum_{j=1}^{n} E\left[w_{i j} \int_{0}^{t} e^{-\alpha(t-u)} f\left(x_{j}(u)\right) d u\right] \\
& +e^{-\alpha t} \frac{1}{\sqrt{n}} \sum_{j=1}^{n} E\left[w_{i j} \int_{0}^{s} e^{-\alpha(s-v)} f\left(x_{j}(v)\right) d v\right] \\
& +\frac{1}{n} \sum_{j=1}^{n} \sum_{k=1}^{n} E\left[w_{i j} w_{i k} \int_{0}^{t} \int_{0}^{s} e^{-\alpha(t-u)} e^{-\alpha(s-v)} f\left(x_{j}(u)\right) f\left(x_{k}(v)\right) d u d v\right] \\
\approx & e^{-\alpha(t+s)}+\frac{1}{n} \sum_{j=1}^{n} E\left[w_{i j}^{2}\right] \int_{0}^{t} \int_{0}^{s} e^{-\alpha(t+s)} e^{\alpha(u+v)} E\left[f\left(x_{j}(u)\right) f\left(x_{j}(v)\right)\right] d u d v \\
= & \left(\text { with, say, } E\left[w_{11}\right]=1\right) e^{-\alpha(t+s)}+e^{-\alpha(t+s)} \int_{0}^{t} \int_{0}^{s} e^{\alpha(u+v)} H_{r}(u, v) d u d v .
\end{aligned}
$$

$H_{r}(u, v)$ stands for $E[f(y) f(z)]$, when $y$ and $z$ are jointly Gaussian random variables with means $e^{-\alpha u}$ and $e^{-\alpha v}$, and correlation $r(u, v)$. We have, also, the boundary conditions $r(t, 0)=r(0, t)=E\left[x_{i}(0) x_{i}(t)\right]=E\left[x_{i}(t)\right]=e^{-\alpha t}$ for all $t \geqq 0$.

In summary, the natural conjecture for (4.1) is that, for any fixed $p$,

$$
\left(x_{1}(\cdot), \cdots, x_{p}(\cdot)\right) \stackrel{w}{\rightarrow} \text { i.i.d. Gaussian processes }
$$

with common mean $\mu(t)=e^{-\alpha t}$, and common correlation $r(t, s)$ satisfying the functional equation

$$
r(t, s)=e^{-\alpha(t+s)}+e^{-\alpha(t+s)} \int_{0}^{t} \int_{0}^{s} e^{\alpha(u+v)} H_{r}(u, v) d u d v
$$

with boundary conditions $r(t, 0)=r(0, t)=e^{-\alpha t}$. In fact, for the special case $f(x)=x$, this is exactly the large system behavior, whether or not $\alpha$ is positive (shown in [5]). But the general case is unproven.

Acknowledgment. Joyce Anderson designed and ran the simulations. It is my pleasure to acknowledge her expert assistance.

\section{REFERENCES}

[1] S. I. AMARI, Characteristics of random nets of analog neuron-like elements, IEEE Trans. Systems Man Cybernet., SMC-2 (1972), pp. 643-657.

[2] H. Chernoff, A measure of asymptotic efficiency for tests of a hypothesis based on the sum of observations, Ann. of Math. Statist., 23 (1952), pp. 493-507.

[3] J. L. FELdmAN AND J. D. CowAN, Large-scale activity in neural nets, II. A model for the brainstem respiratory oscillator, Biol. Cybernet., 17 (1975), pp. 39-51.

[4] S. GemAn, A limit theorem for the norm of random matrices, Ann. of Probab., 8 (1980), pp. $252-261$.

[5] S. Geman AND C. R. HwAng, A chaos hypothesis for some large systems of random equations, Z. Wahrsch. Verw Gebeite, to appear.

[6] S. GemAN AND M. Miller, Computer simulation of brainstem respiratory activity, J. Appl. Physiol., 41 (1976), pp. 931-938.

[7] S. Grossberg, A neural theory of punishment and avoidance, II. Quantitative theory., Math. Biosci., 15 (1972), pp. 253-285. 